\title{
EVALUACIÓN DE LA MICRODUREZA SUPERFICIAL DE DISCOS DE RESINAS PARA RECONSTRUCCIÓN DE MUÑONES. ESTUDIO in vitro
}

\author{
EVALUATION OF THE SURFACE MICRO-HARDNESS OF RESIN DISCS FOR CORE \\ BUILD-UP. IN VITRO STUDY
}

Jesús Alfredo Revollar-Cárdenas', Ana Isabel López-Flores²

\section{RESUMEN}

Objetivo: Comparar, a las 48 horas, la microdureza superficial de las resinas para reconstrucción de muñones Luxacore DMG America, Absolute Dentin Parkell y Z350 XT 3M. Materiales y métodos: El grupo de estudio estuvo conformado por 33 discos de resina, distribuidos en tres subgrupos: dos experimentales cada uno conformado por $(n=11)$, y uno control $(n=11)$. Los dos primeros grupos son resinas de foto activación y el tercero, una resina de activación dual. Estos fueron sometidos al instrumento microdurómetro Vickers, marca LG, en el laboratorio de ensayos mecánicos. Se aplicó una carga de 200 gramos fuerza por 10 segundos para cada indentación. El análisis estadístico se realizó con ayuda del programa SPSS versión 24 y se realizó la prueba de Anova y la prueba de comparación múltiple de Tukey. Resultados: Luego del ensayo de microdureza Vickers (VHN), los resultados fueron: para G1, el valor promedio para la variable microdureza fue 55,24 \pm
1,7 VHN; el G2 arrojó un valor promedio de $54,34 \pm 3,82 \mathrm{VHN}$, y para G3 fue de $75.66 \pm 4.4$ VHN. Conclusión: Las resinas compuestas foto activadas, como material de reconstrucción de muñones sometidos a la prueba de microdureza superficial, mostraron propiedades mecánicas mejoradas en comparación con las resinas duales evaluadas.

Palabras clave: dureza, cementos de resina, polimerización

\section{ABSTRACT}

Objective: Compare the superficial microhardness at 48 hours of the core build-up resins Luxacore DMG America, Absolute Dentin PARKELL and Z350 XT 3M. Materials and methods: The study group consisted of 33 resin discs, distributed in three sub-groups: two experimental groups $(n=11)$ and one control group $(n=11)$. The first two groups were photo activation resins and the third group was a dual activation resin, subjected to

Citar como: Revollar-Cárdenas JA, López-Flores Al. Evaluación de la microdureza superficial de discos de resinas para reconstrucción de muñones. Estudio in vitro. Rev Cient Odontol (Lima). 2018; 6 (1): pp. 29-38

1 Residente de la Especialidad de Rehabilitación Oral, Universidad Científica del Sur. Lima, Perú.

2 Docente de la Especialidad de Rehabilitación Oral, Universidad Científica del Sur. Lima, Perú. 
the Micro-Vickers Hardness Tester - LG Brand in the mechanical testing laboratory. A test force of 200 grams was applied for 10 seconds for each indentation. Statistical analysis was conducted with the help of the SPSS version 24 program and the Anova test and Tukey multiple comparison test were performed.

Results: Following the Vickers microhardness test (VHN), the mean results for the indentations are shown in Table 1 in $\mathrm{VHN}$ values. For $\mathrm{G} 1$ the average microhardness variable was $55.24 \pm 1.7$ VHN. G2 exhibited an average value of $54.34 \pm 3.82 \mathrm{VHN}$. The average microhardness value for G3 was $75.66 \pm 4.4$ VHN. Conclusions: Composite photo activated resins as a core build-up material subjected to surface microhardness testing displayed improved mechanical properties compared to the dual resins evaluated.

Keywords: Hardness, dual-cured resin, resin cements.

\section{INTRODUCCIÓN}

El uso de resinas compuestas ha aumentado considerablemente en los últimos años, sobre todo como respuesta a una mayor demanda de materiales estéticos y de fácil manipulación (1-14). Las resinas compuestas se pueden utilizar en diferentes situaciones clínicas, tales como una restauración directa, indirecta o en la reconstrucción de muñones (2). Los materiales de reconstrucción de muñones son utilizados en la actualidad con mayor frecuencia para proporcionar una morfología ideal para los dientes dañados gravemente, antes de ser preparados para su posterior rehabilitación con restauraciones indirectas cementadas $(1,2,4,6,8,10,13)$.

Los materiales de reconstrucción de muñones a base de resina están disponibles en sus presentaciones de autoactivación, fotoactivación y activación dual. Los materiales de autoactivación tienen la desventaja de requerir un tiempo de polimerización prolongado que no está bajo el control del profesional $(4,10)$. Por otro lado, la colocación de resinas compuestas fotopolimerizadas no permite una mayor reconstrucción de muñones amplios, ya que no se puede asegurar una polimerización adecuada en las partes menos accesibles de las cavidades profundas, donde la luz de polimerización tiene un acceso limitado. Cuando la luz pasa a través de la resina compuesta, se produce una combinación de fenómenos de dispersión, reflexión y absorción en función de la profundidad que reduce la energía de irradiación resultante. Estos efectos dan como resultado una degradación potencial del grado de conversión de monómeros $(10,13)$. La polimerización inadecuada se ha asociado con propiedades mecánicas inferiores, sensibilidad postoperatoria, microfiltración, caries recurrente e irritación de la pulpa causada por monómeros residuales (10).

Una restauración ideal es el objetivo final, pero existen limitaciones al reconstruir los muñones. Entre ellas están el no poder utilizar la técnica a partir de incrementos por el tiempo que demanda el procedimiento y la inseguridad de tener los incrementos de resina polimerizados a pesar de la profundidad de la cavidad $(2,8)$. Por lo tanto, se han desarrollado resinas compuestas duales para este procedimiento de reconstrucción $(1,2)$. El uso de estos materiales resinosos duales permite a los clínicos reconstruir el diente dañado usando capas gruesas de resina, ya que los componentes de autoactivación pueden asegurar la polimerización adecuada, incluso cuando la luz de fotopolimerización es severamente atenuada por la sombra de resina y de la cavidad $(2,3,5$, $8,13,15-23)$. 
Estos materiales han sido diseñados para permitir al clínico reconstruir restauraciones amplias rápidamente en una única capa $(11,12)$. La activación del mecanismo fotopolimerizable conforma rápidamente un contorno rígido de la restauración, mientras que el mecanismo de autoactivación inicia la polimerización en regiones distales a la luz de activación, donde la luz es atenuada e insuficiente como para no lograr una polimerización. Además, se ha afirmado que la velocidad de reacción de polimerización más lenta del mecanismo de autoactivación produce menos tensiones de contracción que el mecanismo de fotoactivación en áreas críticas, como interfaces del material resinoso con tejidos duros dentales y elementos retentivos (por ejemplo, postes de conductos radiculares prefabricados) (8).

Las propiedades físicas, mecánicas y estéticas determinan el comportamiento clínico de los biomateriales y en el caso de las resinas compuestas está íntimamente ligado a su composición química (15). La composición del relleno inorgánico es del que dependen, fundamentalmente, estas propiedades. La dureza es considerada una propiedad mecánica asociada con otras propiedades como la resistencia del material al desgaste, resistencia compresiva, resistencia a la tracción, grado de conversión, que da una indicación general del comportamiento clínico del material $(15,16)$. Mientras que la microdureza es una técnica de ensayo no destructivo que se puede aplicar en el campo de los metales, cerámicas $y$, recientemente, en polímeros. Sirve para relacionar dichos valores con características estructurales de los materiales a ser utilizados $(9,15)$.

El presente estudio comparó a las 48 horas la microdureza superficial de las resinas para reconstrucción de muñones Luxacore DMG America, Absolute Dentin Parkell y Z350 XT 3M (figura 1).

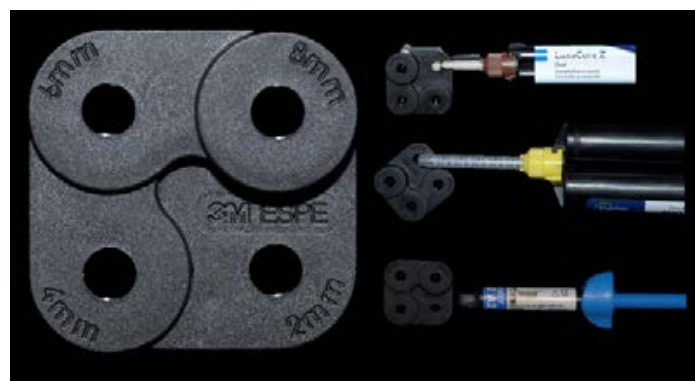

FIGURA 1. RESINAS PARA RECONSTRUCCIÓN DE muñones LuXacore DMG America, Absolute Dentin Parkell y Z350 XT 3M.

\section{MATERIALES Y MÉTODOS}

Este estudio experimental, un ensayo en laboratorio in vitro, fue realizado en el laboratorio de ensayos mecánicos de la Carrera de Estomatología de la Universidad Científica del Sur, en el período de abril y diciembre de 2017. La muestra consistió en 33 discos de resina compuesta, distribuidos en tres subgrupos: dos experimentales, cada uno conformado por $(\mathrm{n}=11)$, y uno control $(n=11)$, siendo los dos primeros grupos resinas de fotoactivación y el tercero, una resina de activación dual (figura 2). Estos grupos fueron sometidos al instrumento microdurómetro Vickers, marca LG, en el laboratorio de ensayos mecánicos. Se empleó una carga de 200 gramos fuerza durante 10 segundos para cada indentación (figura 3). La investigación fue presentada, para su revisión y aprobación, a la Comisión de Revisión de Aspectos Metodológicos y Éticos de la carrera

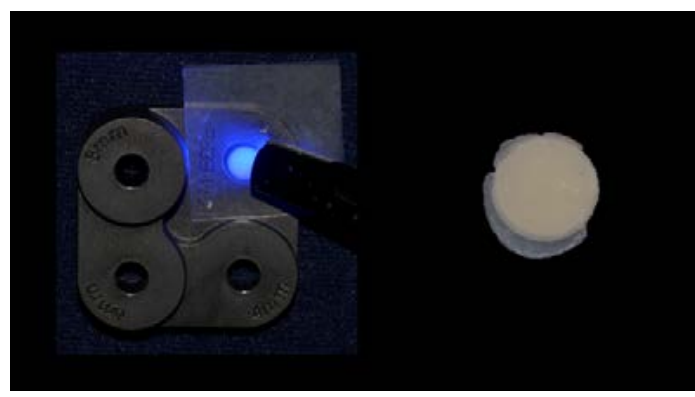

FIGURA 2. FABRICACIÓN DE DISCOS DE RESINA DE FOTOACTIVACIÓN Y ACTIVACIÓN DUAL DE 2 MM DE ESPESOR POR 5 MM DE DIÁMETRO. 


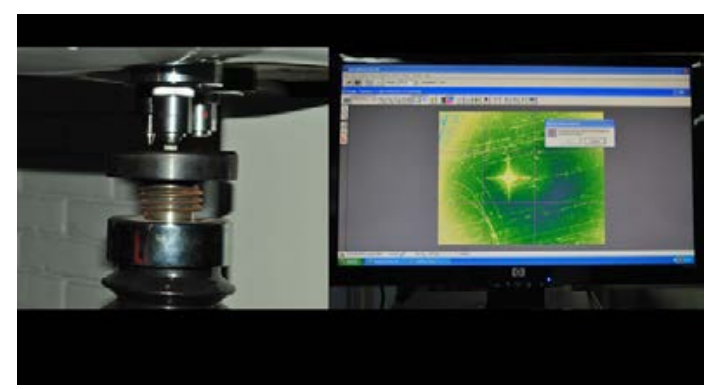

Figura 3. MicrodurómETRO VicKERS (MARCA

LG) EN EL LABORATORIO DE ENSAYOS MECÁNICOS. ESPECÍMENES SOMETIDOS A UNA CARGA DE 200 GRAMOS FUERZA DURANTE 10 SEGUNDOS.

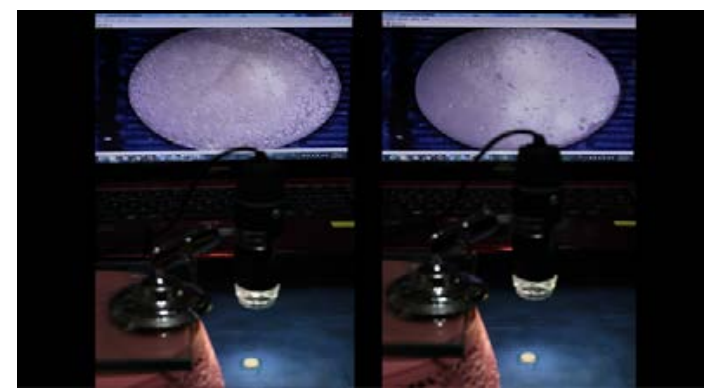

FIGURA 4. LUEGO DE REALIZAR EL PULIDO DE LA SUPERFICIE DE LOS DISCOS DE RESINA ANTES DE LA INDENTACIÓN, SE VERIFICA EL GRADO DE PULIDO USANDO UN MICROSCOPIO DIGITAL.

de Estomatología de la Universidad Científica del Sur.

Se utilizó la fórmula para estimar una proporción, a un nivel de confianza del $95 \%$, con un margen de error del $5 \%$, lo que dio como resultado 33 discos de resina compuesta y los criterios de exclusión fueron: discos con burbujas, discos con fracturas o fisuras, discos sin pulido (figura 4). Las resinas para reconstrucción de muñones que se utilizaron en el estudio se obtuvieron a través de los distribuidores autorizados para las diferentes marcas, con fecha de vencimiento no menor a diciembre de 2017 (figura 5).

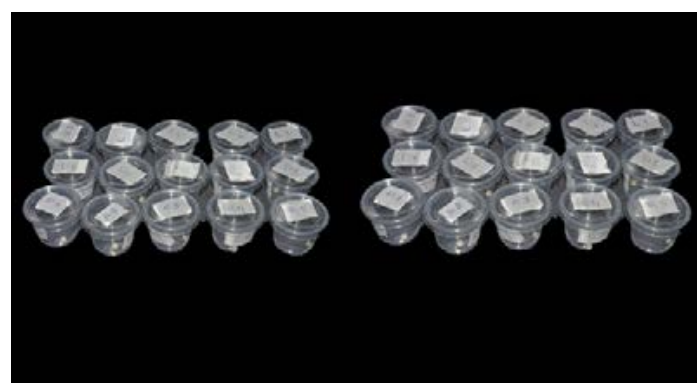

Figura 5. Almacenamiento de los especímenes POR 48 HORAS A TEMPERATURA AMBIENTE.

El análisis estadístico se realizó con ayuda del programa SPSS versión 24 . Este comenzó mediante la estadística descriptiva que consistió en la presentación de las medidas de tendencia central (media y mediana) y las medidas de dispersión (desviación estándar, mínimo, máximo, rango, varianza) de la variable microdureza en cada grupo de estudio: grupo 1 (Luxa Core), grupo 2 (Absolut Dentin) y grupo 3 (z350 XT 3M). Luego, se evaluó la normalidad en los grupos de estudio mediante la prueba de Shapiro Wilk. Finalmente, se realizaron las comparaciones entre grupos con las pruebas estadística de Anova y la prueba de comparación múltiple de Tukey. Todo se trabajó con un nivel de significancia de $p<0,05$.

\section{RESULTADOS}

Luego del ensayo de microdureza Vickers (VHN) se encontraron diversos

Tabla 1. Evaluación de La Microdureza (VHN) DE TRES RESINAS PARA RECONSTRUCCIÓN DE MUÑONES

\begin{tabular}{|l|c|c|c|c|c|}
\hline Marca de resina & N. & Media & D.E & Min & Max \\
\hline Luxa Core & 11 & 55,24 & 1,7 & 52,8 & 57,1 \\
\hline Absolute Dentim & 11 & 54,34 & 3,82 & 51,5 & 59,7 \\
\hline Z- 350 & 11 & 75,66 & 4,4 & 71,3 & 86,6 \\
\hline
\end{tabular}

$p<0,001$ Prueba de Anova 
TABLA 2. Comparación de LA SIGNIFICANCIA ESTADístiCA DE LA MICROdUREZA ENTRE LAS 3 RESINAS EVALUADAS

\begin{tabular}{|c|c|c|c|c|c|c|c|c|}
\hline \multirow{3}{*}{$\begin{array}{c}\text { Marca de } \\
\text { resina }\end{array}$} & \multicolumn{4}{|c|}{ Luxa Core } & \multicolumn{4}{|c|}{ Absolute Dentim } \\
\hline & \multirow{2}{*}{$\begin{array}{l}\text { Dif. } \\
\text { medias }\end{array}$} & \multicolumn{2}{|c|}{$\begin{array}{l}\text { 95\% Intervalo } \\
\text { de confianza }\end{array}$} & \multirow[t]{2}{*}{$P$} & \multirow{2}{*}{$\begin{array}{c}\text { Dif. } \\
\text { medias }\end{array}$} & \multicolumn{2}{|c|}{$\begin{array}{l}95 \% \text { Intervalo } \\
\text { de confianza }\end{array}$} & \multirow[t]{2}{*}{$P$} \\
\hline & & LI & LS & & & LI & LS & \\
\hline Z-350 & 25,17 & 20,9 & 29,36 & $<0,001$ & 23,9 & 19,67 & 28,12 & $<0,001$ \\
\hline Luxea Core & & & & & $-1,23$ & $-5,46$ & 2,99 & 0,753 \\
\hline
\end{tabular}

Prueba de comparación múltiple de Tukey

valores para cada grupo de estudio que se anotaron en la ficha de recolección de datos, según cada tipo de resina. Los resultados promedio de las indentaciones se muestran en la tabla 1, tanto en valores de $\mathrm{VHN}$ : para $\mathrm{G} 1$, el valor promedio para la variable microdureza fue 55,24 \pm 1,7 VHN; el G2 arrojó un valor promedio de $54,34 \pm 3,82 \mathrm{VHN}$; para G3 fue de 75,66 $\pm 4,4 \mathrm{VHN}$. El valor máximo para el G1 fue de 57,1 VHN mientras que el valor mínimo fue de 52,8 VHN. El valor máximo para el grupo 2 fue de 59,7 VHN, mientras que el valor mínimo fue de 51,5 VHN. El valor máximo para el grupo 3 fue de $86,6 \mathrm{VHN}$, mientras que el valor mínimo fue de 71,3VHN. Los resultados para cada muestra, según la marca de resina, se muestran en la tabla 1.

El análisis bivariado se realizó con la prueba de ANOVA para muestras independientes. Se encontraron diferencias estadísticamente significativas $(p<0,05)$ entre todos los grupos estudiados, ejecutándose entonces el test de comparaciones múltiples de Tukey (tabla 2). Se encontraron diferencias estadísticamente significativas entre el G3 y G1, mientras que entre $\mathrm{G} 1$ y $\mathrm{G} 2$ existieron diferencias, pero que no fueron estadísticamente significativas. Las diferencias encontradas entre $\mathrm{G} 3$ y $\mathrm{G} 1$ y entre $\mathrm{G} 3$ y G2 sí fueron estadísticamente significativas. Los mayores valores de microdureza superficial se encontraron en G3, cuyas diferencias fueron estadísticamente significativas comparadas con G1y G2.

\section{DISCUSIÓN}

Las propiedades mecánicas de las resinas para reconstrucción de muñones contribuyen a su comportamiento clínico, por lo que se han realizado diversos estudios a este nivel; sin embargo, su dureza no ha sido ampliamente estudiada. Por esta razón, el propósito de la presente investigación fue comparar la microdureza de tres resinas para reconstrucción de muñones y observar si los valores que presentan estos biomateriales difieren unos de otros. Los estudios que anteceden al presente (1) demuestran que los valores de microdureza encontrados para la resina $Z 350$ de $3 \mathrm{M}$ son altos; además, existe mucha literatura acerca de esta resina para reconstrucción de muñón, por lo que en este estudio ha sido considerado como el grupo control (2). Las otras dos resinas para reconstrucción de muñones tuvieron participación en diversos estudios comparativos, a excepción de Absolute Dentin Parkell, que es un material relativamente nuevo $(3,4)$.

Se realizó un estudio piloto previo para determinar el tamaño muestral, utilizando un número de especímenes de prueba que se equipara al utilizado en los estudios previos. Todos los especímenes de prueba fueron preparados de manera padronizada, según las indicaciones del fabricante, y se contó con todos los criterios de inclusión, previa capacitación deloperador(2-4).Lasmuestras fueron evaluadas meticulosamente 
por el operador con un microscopio digital anexado a la computadora, para observar que presente un pulido ideal para su posterior indentación; luego, fue evaluado por un ingeniero con el microscopio para realizar la indentación en una zona libre de microburbujas. Antes del ensayo de microdureza, los especímenes fueron almacenados a $24{ }^{\circ} \mathrm{C}$ (temperatura ambiente), a diferencia de otros estudios anteriormente realizados, que lo ejecutaron a $37{ }^{\circ} \mathrm{C}$ (temperatura corporal) (1, 5-7). Sin embargo, hay estudios que utilizaron los criterios de almacenaje similares al nuestro $(7,8)$. El ensayo de microdureza fue realizado por un ingeniero mecánico capacitado y con experiencia en el manejo del microdurómetro Vickers, marca LG, en el laboratorio de ensayos de la Universidad Científica del Sur.

La microdureza ha sido directamente relacionada con el grado de conversión de los polímeros $(5,9)$. Indicamos entonces, de manera indirecta, que a mayor valor de microdureza de una resina para reconstrucción de muñones, el comportamiento del material es mejor. Esto repercute de manera favorable en una mejor compatibilidad biológica en relación con el complejo dentinopulpar, porque el porcentaje de monómeros convertidos es mayor y, además, brinda un mejor sellado marginal contra la nano y microfiltración $(10,11)$. Por otro lado, las resinas para la reconstrucción de muñones, a menudo, se requieren para reconstruir y proporcionar una morfología ideal para los dientes dañados gravemente, antes de la preparación para restauraciones indirectas cementadas. $Y$ si bien es cierto que la reconstrucción estará cubierta por la restauración indirecta, debe tener la dureza ideal para evitar una deformación plástica que comprometa no solo su biocompatibilidad y el sellado con lo que resta del muñón, sino su permanencia en la cavidad oral. Los resultados conse- guidos en esta investigación indican que existen diferencias entre cada una de las resinas para reconstrucción de muñones estudiadas; sin embargo, no todas son estadísticamente significativas.

Los valores encontrados en el grupo control (G3) fueron más altos con respecto a los valores de G1 y G2. Esto puede ser provocado por las diferencias en la forma de polimerización: mientras que el grupo control se fotoactiva, los grupos restantes tienen una activación dual. Asimismo, cabe indicar que la composición de la matriz también es diferente: la resina Z350 XT contiene resinas bis-GMA, UDMA, TEGDMA y bis-EMA, para controlar la contracción; PEGDMA fue sustituida por una porción de resina TEGDMA en $3 \mathrm{M}^{\mathrm{TM}}$ Filtek $^{\mathrm{TM}}$ Z350 XT; mientras que G1 y G2 contienen resina acrílica, polvo de vidrio y sílice, dimetacrilato de uretano, dimetacrilato alifático y dimetacrilato aromático $(3,4,14)$, lo que podría explicar la menor dureza de G1. Igualmente, los iniciadores químicos también difieren. Mientras que G3 contiene canforoquinona, G1 y G2 inician su reacción de autopolimerización a través de una reacción redox, que tiene como iniciador al peróxido de benzoilo (PB), y como activador una amina aromática, por ejemplo, N-dimetilp-toluidina $(12,13)$.

Las diferencias encontradas entre $\mathrm{G} 1$ y G2, no fueron estadísticamente significativas, lo que podría deberse a que la forma de polimerización es la misma, y su matriz de resina está basada en metacrilato; por tanto, se podría indicar que el comportamiento mecánico es similar $(2,3,4,14,15)$.

Por otro lado, las diferencias entre G3 y G1 sí tuvieron significancia estadística. Una de las razones podría ser el contenido y tipo de relleno inorgánico. La resina G3 no solo contiene un porcentaje de relleno mayor que $\mathrm{G} 1$ y $\mathrm{G} 2(12,13,16)$, 
también contiene partículas de zirconio $(2,47)$, lo que podría explicar su mayor dureza, pues $\mathrm{G} 1$ y $\mathrm{G} 2$ tienen polvo de vidrio y sílice $(4,14)$. Otra de las razones podría ser la naturaleza de la matriz, ya que el bis-GMA, monómero principal de G3, es una molécula muy grande y de alto peso molecular, que genera un polímero complejo y tridimensional de altas propiedades físicas y mecánicas en comparación con los metacrilatos (17), monómero principal de G1 y G2, que tiene una estructura más simple que el bis-GMA y un peso molecular más bajo. Esto podría significar polímeros con propiedades mecánicas menores (18), lo que explicaría, además, los valores bajos de microdureza que difieren estadísticamente de todos los grupos estudiados. En este sentido, podría indicarse que el comportamiento mecánico de la resina Z350 XT (G3) sería el ideal comparado con las otras resinas duales que tienen menor grado de dureza, lo que significaría que un menor grado de conversión, como se ha mencionado, optimiza la biocompatibilidad y el sellado marginal $(10,7)$. Sin embargo, los valores de microdureza encontrados para G1 y G2 se encuentran dentro de los valores aceptados $(3,4,18)$.

Al ser comparada la microdureza de G1 y G2, se obtuvo mayores valores para G1, resina dual, lo que podría deberse a la mayor cantidad de metacrilatos que presenta $(3,4,14)$. Sin embargo, las diferencias no fueron estadísticamente significativas, por lo que el comportamiento mecánico sería similar. Cabe mencionar que $\mathrm{G} 1$ tiene un adhesivo dual con el que se utiliza el biomaterial, que es Luxa Bond Total Etch, un medio adhesivo de activación dual para esmalte y dentina concebido para ser utilizado principalmente con resinas de activación dual (19). A diferencia de $\mathrm{G} 2$, indica que puede ser utilizado con todos los agentes de unión de esmalte/dentina compatibles con resinas duales, incluidos los productos de unión basados en 4-Meta de Parkell (Brush\&Bond $^{\mathrm{TM}}$, Touch\&Bond $($, Amalgambond $^{\mathrm{TM}}, \quad$ C\&B-Metabond $\AA \quad \mathrm{y}$ SEcure $\left.{ }^{\mathrm{TM}}\right)$. También es compatible con los productos Add\&Bond ${ }^{\mathrm{TM}}$, DuraFinish ${ }^{\mathrm{TM}}$ y DuraFinish ${ }^{\mathrm{TM}}$ All-Cure de Parkell.

En general, los mayores valores de microdureza se encontraron en los cuerpos de prueba de G3, con diferencias estadísticamente significativas solo al ser comparados con $\mathrm{G} 1$ y G2. Los menores valores se encontraron en los cuerpos de prueba de G3, cuyas diferencias con el otro G2 no fueron estadísticamente significativas. Estos resultados podrían deberse al tipo y porcentaje de relleno, la naturaleza de la matriz resinosa y el grado de conversión.

Los últimos estudios sobre microdureza comparan materiales exclusivamente de restauración. Pocas investigaciones se han encontrado sobre microdureza de resinas para reconstrucción de muñones; sin embargo, se ha tratado que, por lo menos, la metodología utilizada para la fabricación de los especímenes de prueba de la presente investigación, sea muy similar a la utilizada en las investigaciones previas. Es así como Galvão y col. (14) utilizaron en su estudio una metodología y tamaño muestral similares a los de la presente investigación para evaluar la microdureza de las resinas duales y el cemento dual. A diferencia de nuestra investigación, se estudiaron resinas duales Luxa Core DFGM y Absolut Dentin Parkell, con una resina de fotoactivación Z350 XT de 3M. Pero los resultados hallados de la prueba de microdureza fueron muy semejantes a los encontrados en el presente estudio, a pesar de que la carga aplicada y el tiempo de exposición a la indentación fueron diferentes. La luz también fue diferente.

Otra investigación (22) comparó la efectividad de la autoactivación y la fotoactivación en la polimerización de 
materiales para reconstrucción de muñones duales, de las marcas Clearfil DC Core Automix (Kuraray, Tbkyo), flujo Core'X (Dentsply DeTrey, Konstanz, Alemania), LuxaCore Z-Dual (DMG, Hamburgo, Alemania) y MultiCore Flow (Ivoclar Vivadent, Schaan, Liechtenstein). El tamaño muestral y la confección de los cuerpos de prueba fue muy similar al utilizado en el presente estudio. Del mismo modo, el almacenamiento de los cuerpos de prueba antes de realizar las indentaciones se hizo a temperatura ambiente, tal como se almacenaron las muestras de nuestra investigación. La diferencia con nuestro estudio fue la medición en KHN. Los resultados obtenidos en el presente estudio fueron que todos los otros materiales exhibieron una disminución significativa de KHN a profundidades mayores en solo uno de los seis grupos fotoactivados; esto determina que, a pesar de la activación a diferentes profundidades, se tiene la seguridad de que las resinas duales se activarán y mantendrán sus propiedades mecánicas ideales. Sin embargo, también nos muestra que, al ser una resina exclusivamente de fotoactivación, podría presentar falta de polimerización en las zonas más profundas de la cavidad por presentarse atenuada la fotoactivación, tomando como protocolo la colocación por incrementos no mayores a $2 \mathrm{~mm}$, como se indica en diferentes investigaciones $(21,22)$.

Otros autores (23) evaluaron el grado de conversión y microdureza Vickers de RelyX ${ }^{\mathrm{TM}}$ Unicem con las siguientes variables la distancia y el tiempo de polimerización, la interposición de un disco de cerámica y la polimerización química únicamente. Para cada grupo fueron diferentes los valores obtenidos de microdureza, con valores más altos en el grupo de mayor tiempo de polimerización sin interposición del disco de cerámica. Sin embargo, los resultados obtenidos en la presente investigación, al ser comparados, se encontraron mucho más bajos. Esto tendría una relación con la potencia de luz utilizada para la confección de los especímenes de prueba, que fue mucho menor a la utilizada en nuestra investigación; además que el tiempo y carga para el ensayo de indentación fueron diferentes también. Por otro lado, este estudio corrobora que los valores de microdureza para la polimerización química son menores que los obtenidos con la polimerización dual. En la confección de los especímenes de prueba para la presente investigación, se tomó en cuenta ambos modos de polimerización, ya que se dejó un tiempo para la activación química y, luego, se procedió con la fotoactivación, tratando de conseguir de esta forma grados de conversión y valores de microdureza más elevados.

Se concluye, con base en los resultados obtenidos en la investigación, que la resina para reconstrucción de muñones Z350 XT 3M presenta un mejor comportamiento mecánico comparado con las otras resinas para reconstrucción estudiadas, por presentar mayores valores de dureza. Por otro lado, las resinas duales para reconstrucción de muñones Luxa Core DFGM y Absolute Dentin Parkell presentan propiedades mecánicas menores en relación con la resina de foto activación por presentar valores de dureza inferiores. Sin embargo, el uso de resinas duales como materiales para reconstrucción de muñones asegura valores de microdureza confiables incluso en regiones profundas, donde no hay luz disponible por el tipo de activación que presenta; además, se encuentran dentro de los valores permitidos $(5,6)$.

Por último, teniendo en cuenta las limitaciones del presente estudio, se puede concluir que los resultados obtenidos rechazan la hipótesis nula, dado que se encontraron diferencias entre los valores 
de todas las resinas duales para reconstrucción de muñones, aunque en algunos casos no fueron estadísticamente significativas, y que los valores obtenidos para la Resina Z350 XT de 3M es mayor que los demás grupos. Por su parte, los grupos de las resinas duales Luxa Core DFGM y Absolute Dentin Parkell, al ser comparadas, no mostraron diferencias estadísticamente significativas.

\section{CONCLUSIONES}

La resina compuesta fotoactivada, como material de reconstrucción de muñón, presentó los valores más altos de microdureza superficial, lo que indica propiedades mecánicas mejoradas comparadas con las resinas duales evaluadas. Sin embargo, la resina dual, como material para reconstrucción de muñones, es una buena opción en algunos casos clínicos, ya que los valores encontrados están por encima del mínimo requerido.

\section{REFERENCIAS BIBLIOGRÁFICAS}

1. Hofmann N, Papshart G, Hugo B, Klaiber B. Comparison of photo-activation versus chemical or dual-curing of resin-based luting cements regarding flexural strength modulus and surface hardness. J Oral Rehab. 2001; 28: 1022-8.

2. Galvão CA, De Cerqueira A. Effect of curing mode on the hardness of dual-cured composite resin core build-up materials. Braz Oral Res. 2010; 24 (2): 245-9.

3. D'Arcangelo C, Cinelli M, De Angelis F, D'Amario M. The effect of resin cement film thickness on the pullout strength of a fiber-reinforced post system. J Prosthet Dent. 2007; 98 (3): 193-8.

4. Karakis D, Zeynep A, Dogan A. Effect of self and dual-curing on degree of conversion and crosslink density of dual-cure core build-up materials. J Prosthodont Res. 2017; 61 (2): 210-6.

5. Pereira SG, Fulgêncio R, Nunes TG, Toledano M, Osorio R, Carvalho RM. Effect of curing protocol on the polymerization of dual-cured resin cements. Dent Mater. 2010; 26 (7): 710-8.

6. Tauböck TT, Bortolotto T, Buchalla W, Attin T, Krejci I. Influence of light-curing protocols on polymerization shrinkage and shrinkage force of a dual-cured core build-up resin composite. Eur J Oral Sci. 2010; 118 (4): 423-9.

7. Silva T, Coutinho M, Cardozo P, Silva L, Zorzatto J. Conventional dual-cure versus self-adhesive resin cements in dentin bond integrity. J Appl Oral Sci. 2011; 19 (4): 355-62.

8. Kournetas N, Tzoutzas I, Eliades G. Monomer conversion in dual-cured core buildup materials. Oper Dent. 2011; 36 (1): 92-97.

9. Ruiz JM, Ceballos L, Fuentes M, Osario R, Toledano M, García-Godoy F. Propiedades mecánicas de resinas compuestas modificadas o no con poliácidos. Av Odontoestom. 2003; 19 (6): 291-7. 
10. Tauböck TT, Bortolotto T, Buchalla W, Attin T, Krejci I. Comparing the effectiveness of self-curing and light curing in polymerization of dual-cured core buildup materials. JADA 2011;142 (8): 950-6.

11. Bitter K, Schubert A, Neumann K, Blunck E, Sterzenbach G, Rüttermann S. Are self-adhesive resin cements suitable as core build-up materials? Analyses of maximum load capability, margin integrity, and physical properties. Clin Oral Invest. 2016; 20: 1337-45.

12. Migliau G, Piccoli L, Di Carlo S. Comparison between three glass fiber post cementation techniques. Ann di Stomat. 2017; 8 (1): 29-33.

13. Keiichi $Y$, Xiangfeng M. Influence of light-exposure methods and depths of cavity on the microhardness of dual-cured core build-up resin composites. J Appl Oral Sci. 2014; 22 (1): 44-51.

14. Kurzer M. Estudio comparativo de dureza en dientes artificiales fabricados con diferentes tipos de resinas acrílicas. Rev Eia 2006; 6: 121-8.

15. Phillips. Ciencia de los materiales dentales. Madrid: Elsevier; 2004.

16. Macchi R. Materiales dentales. Buenos Aires: Médica Panamericana; 2007.

17. Menezes MA, Muench A. Dureza Knoop de resinas compostas em funcao do numero relativo de radicais libres. Rev Odont Univ São Paulo 2006; 12: 281-5.

18. Santerre JP, Shalii L, Leung BW. Relation of dental composite formulations to their degradation and the release of hydrolyzed polymeric-resin-derived products. Rev Oral Biol Med. 2001; 12 (2): 136-51.

19. Ramires E, Codargo I, Vaz CR. Caracterización metalográfica de aleaciones metálicas utilizadas como biomateriales en implantes quirúrgicos. Univ Est Paul. 2002; 21-4.

20. Spinell T, Schedle A, Watts DC. Polymerization shrinkage kinetics of dimethacrylate resin-cements. Dent Mater J. 2009; 25 (8): 1058-66.

21. Cekic-Nagas I, Ergun G, Vallittu PK, Lassila LV. Influence of polymerization mode on degree of conversion and micropush-out bond strength of resin core systems using different adhesive systems. Dent Mater J. 2008; 27 (3): 376-85.

22. Hervás J, Martínez M, Cabanes J, Barjau A, Galve P. Resinas compuestas. Revisión de los materiales e indicaciones clínicas. Med Oral Patol Oral Cir. Bucal. 2006; 11 (2): 216-7.

23. Camargo E, Moreschi E, Baseggio W, Cury JA, Pascotto RC. Composite depth of cure using four polymerization techniques. J Appl Oral Sci. 2009; 17: 446-50. 